\title{
El Clima desértico costero con nublados abundantes del desierto de Atacama y su relación con los recursos naturales energía solar y agua de niebla. Caso de estudio Alto Patache $\left(20,5^{\circ} \mathrm{S}\right)$, región de Tarapacá, Chile ${ }^{1}$
}

\author{
Pablo Osses², Rodrigo Escobar ${ }^{3}$, Camilo del Rio ${ }^{4}$, Redlich Garcia ${ }^{5}$ \\ y Constanza Vargas 6
}

\begin{abstract}
RESUMEN
Considerando los crecientes requerimientos de agua y energía en zonas áridas, se aborda la presencia del estratocúmulo costero como potencial recurso hídrico y el comportamiento de la radiación solar como fuente de energía. Este estudio exploratorio busca conocer las relaciones entre atenuación de la irradiancia por tipología de niebla (orográfica o advectiva) y la producción de agua de niebla asociada. Se analizó septiembre 2013 mediante imágenes satelitales GOES, datos del neblinómetro (SFC) y el radiómetro localizados en la Estación Atacama UC_Oasis Niebla Alto Patache $\left(20^{\circ} 49^{\prime} \mathrm{S}-70^{\circ} 09^{\circ} \mathrm{O}-800 \mathrm{~m}\right.$ s.n.m.). Se encontró complementariedad entre los ciclos diarios de estos recursos y sus horarios productivos de agua y energía (noche y día) respectivamente. Esta investigación motiva estudios posteriores sobre agua de niebla y energía solar como recursos complementarios en línea con implementar tecnologías para tratamiento, manejo y distribución del agua, así como limpieza de paneles solares.
\end{abstract}

Palabras claves: Irradiancia $\left(\mathrm{W} / \mathrm{m}^{2}\right)$, niebla orográfica y advectiva, recursos naturales renovables

\begin{abstract}
Considering the increase of water and energy requirements in arid zones, this work combine the presence of the coastal stratocumulus as a potential water resource and the behavior of solar radiation as an energy source. The aim is to know the relationships between attenuation of irradiance by fog typology (orographic or advective) and the yields of associated fog water. September 2013 was analyzed using GOES satellite images, data from the Standard Fog Collector (SFC) and the radiometer located at the Atacama UC Station, Oasis de Niebla Alto Patache ( $20^{\circ} 49^{\prime} \mathrm{S}-70^{\circ} 09^{\prime} \mathrm{O}-800 \mathrm{~m}$ a.s.l.). Results show complementarity between the daily cycles of these resources and their productive hours of water and energy (night and day) respectively. This research motivates subsequent studies on fog water and solar energy as complementary resources in line with implementing technologies for water treatment, management and distribution, as well as solar panel dust cleaning.
\end{abstract}

Key words: irradiance $\left(\mathrm{W} / \mathrm{m}^{2}\right)$, Advective and Orographic Fog, Renewable Natural Resources.

Los autores quieren agradecer a Natalia Osorio por sus aportes en terreno durante los inicios de este trabajo. Artículo recibido el 30 de marzo de 2017, aceptado el 30 de agosto de 2017 y corregido el 30 de octubre de 2017.

Centro UC Desierto de Atacama / Instituto de Geografía, Pontificia Universidad Católica de Chile (Chile). E-mail: posses@uc.cl

Centro UC de Energía y Centro UC Desierto de Atacama / Escuela de Ingeniería, Pontificia Universidad Católica de Chile (Chile). E-mail: rescobar@ing.puc.cl

Centro UC Derecho y Gestión de Aguas, y Centro UC Desierto de Atacama / Instituto de Geografía, Pontificia Universidad Católica de Chile (Chile).E-mail: cdelriol@uc.cl

Departamento de Ingeniería Mecánica y Metalurgia, Pontificia Universidad Católica de Chile (Chile). E-mail: rjgarcia2@uc.cl

Centro UC Desierto de Atacama / Instituto de Geografía, Pontificia Universidad Católica de Chile (Chile). E-mail: cgvarga1@uc.cl 
En el desierto de Atacama existen dos recursos fundamentales para el ser humano en su condición de existencia y desarrollo económico productivo, los recursos hídricos y la generación de energía (Parrado et al., 2016). La escasez de agua por un lado y las fuentes de energía basadas en combustibles fósiles por otra, generan una creciente problemática para este territorio extremo, ambientalmente frágil y que busca opciones sustentables para su desarrollo presente y futuro. Es así como en el presente artículo se analizan dos recursos abundantes del desierto costero y poco explorados en cuanto a su interacción, como alternativa o complemento a las fuentes de agua y energía utilizados en la actualidad.

En materia de agua dulce superficial disponible para el consumo, Chile tiene una disponibilidad de $53.000 \mathrm{~m}^{3} /$ persona/año, muy por encima de la media mundial de $6.600 \mathrm{~m}^{3} /$ persona/ año. Sin embargo, desde la Región Metropolitana al norte, el promedio de agua disponible es de $800 \mathrm{~m}^{3} /$ persona/año, siendo que el mínimo recomendado para un desarrollo sostenible es de $2.000 \mathrm{~m}^{3} /$ persona/año (Banco Mundial, 2011: 5). Dada esta problemática, la exploración de fuentes alternativas y complementarias de agua dulce en el Norte de Chile es ineludible. De esta forma, el agua de niebla puede contribuir a moderar la escasez de agua en el Norte Grande (CIDERH, 2013).

Por otra parte, el desarrollo económico, industrial y tecnológico en el país ha suscitado otra gran demanda, la energía. Chile ha trabajado por tener un balance estable y confiable de fuentes de energía para satisfacer sus crecientes necesidades (Agostini et al., 2015: 40). Dentro de las fuentes de energía que han utilizado, destacan las hidroeléctricas, gas natural, carbón y petróleo. Sin embargo, el año 2013, se publicó la Ley 20.698, que establece que al año 2025, el $20 \%$ de la energía comercializada debe provenir de fuentes renovables no convencionales (Santana et al., 2014: 6). Chile es considerado un país muy atractivo para desarrollar e implementar tecnologías de este tipo dada su ubicación geográfica y gran diversidad biológica, proporcionando abundantes recursos energéticos (Agostini et al., 2015: 40). El desierto de Atacama tiene uno de los mayores potenciales solares del mundo, con casi 365 días de cielo despejado, alta radiación solar y baja humedad, proporcionando una importante oportunidad para contribuir a la diversificación energética del país (Agostini et al., 2015: 40). Un estudio realizado por ChileRenovables indica que el desierto de Atacama es la región con mayor radiación solar del mundo $\left(275 \mathrm{~W} / \mathrm{m}^{2}\right)$. A su vez es sabido que la mayor atenuación de la radiación solar es producida por la presencia de una cobertura nubosa (Kleissl, 2013) por lo tanto resulta de gran interés conocer las implicancias de los nublados abundantes del desierto costero.

El aprovechamiento del agua de niebla como fuente alternativa ha sido estudiada y utilizada con distintos fines tanto en Chile como en diversos países y climas del mundo entero. La producción exitosa de agua de niebla y su continuidad en el tiempo depende de las condiciones y tipo de niebla (frecuencia, contenido de agua, velocidad de viento, etc.) y en buena medida de la participación e involucramiento de las comunidades locales (Klemm et al., 2012: 226-227). Estudios preliminares han estimado que es un recurso abundante y sub-explotado (Larraín et al., 2002; Cáceres et al., 2007; Cereceda et al., 2008a). Sin embargo, en cuanto al progreso de la evaluación de recurso solar reportada para Chile se indica que está dotado con un de los más altos niveles radiación solar del mundo (Escobar et al., 2015: 146). Los datos de radiación solar para grandes regiones espaciales se pueden obtener a partir de redes de estaciones terrestres, que proporcionan puntos de datos discretos a partir de los cuales se puede obtener un mapa continuo por medio 
de un esquema de interpolación adecuado (Ortega et al., 2010). El clima desértico costero con nublados abundantes (Weischet, 1975: 368) está determinado principalmente por la presencia del anticiclón del Pacífico, condicionando las prácticamente nulas precipitaciones $(0,6 \mathrm{~mm}$ promedio anual en la ciudad de lquique) (Dirección Meteorológica de Chile, 2017). La subsidencia de aire cálido propia de estas latitudes produce una inversión térmica, generando una primera capa marina húmeda y fría, intensificada por la corriente de Humboldt y las surgencias de aguas profundas. La humedad bajo ciertas condiciones condensa, formando una extensa nube del tipo estratocúmulo (Sc), la cual al entrar en contacto con la costa forma la niebla o camanchaca. En esta zona es posible reconocer dos tipos de niebla, advectiva y orográfica, las primeras son transportadas por el viento hacia la costa con una altitud entre 500 y 1.000 metros y un grosor de 300 metros en promedio (Cereceda et al., 2002: 261; Osses et al., 2005: 135). Por su parte, la niebla orográfica se forma por el ascenso de una masa de aire húmedo por efecto del relieve, en este caso el farellón costero de la cordillera de la Costa.

La presencia de nube Sc y la niebla en el área de estudio presentan un ciclo anual bien definido con menor presencia desde diciembre a abril y mayor presencia desde mayo a noviembre (Cereceda et al., 2008a y 2008b; Farías et al., 2005), asimismo hay un ciclo diario predominante, con mayor presencia durante la noche y amanecer y durante el día es generalmente disipada y evaporada por la radiación solar.

La evaluación del recurso solar en Chile data de la década de 1960, cuando la Universidad Técnica Federico Santa María realizó esfuerzos recopilando datos de alrededor de 90 piranógrafos y dispositivos Stokes - Campbell, abarcando un período de aproximadamente 20 años. La mayoría de estos datos tienen un nivel de incertidumbre relativamente grande, propia de los sensores obsoletos de la época, lo que hace que dicha data sea inadecuada para la planificación energética a nivel de política nacional. Sin embargo, los datos medios mensuales se consideran útiles para las aplicaciones de calentamiento solar de agua, y están disponibles (Sarmiento, 2009; CNE/PNUD/ UTFSM, 2008).

Así, el presente trabajo busca analizar y caracterizar la presencia de niebla costera en su ciclo diario y su relación con la radiación solar, distinguiendo las tipologías de nieblas y su productividad de agua. Las preguntas de investigación que guían este trabajo, tales como, ¿Qué tipología de niebla atenúa más la radiación?, ¿Es la nubosidad abundante un impedimento para el aprovechamiento de la energía solar?, buscan establecer la factibilidad de utilizar energía solar y agua de niebla de manera complementaria en un contexto de escasez de ambos recursos fundamentales, esto dado principalmente por la atenuación en la radiación producto de la nubosidad, situación que podría llevar a una eventual anulación de un recurso en función del otro (agua y energía). Es en este sentido que explorar el comportamiento de ambos recursos puede ser relevante para futuros desarrollos, especialmente en el desierto de Atacama donde la presión por energía y recursos hídricos es creciente.

La investigación se desarrolla en la Estación Atacama UC Oasis Niebla Alto Patache $\left(20^{\circ} 49^{\prime} \mathrm{S}\right.$ $-70^{\circ} 09^{`}$ O), donde el instrumental se localiza sobre el farellón costero, a 850 m s.n.m. y distantes aproximados $4 \mathrm{~km}$ de la línea de costa (Figura $\mathrm{N}^{\circ} 1$ ). 
Figura No 1

Estación Atacama UC, Oasis de Niebla Alto Patache (2049’ S - $\left.70^{\circ} 09^{`} \mathrm{O}\right)$

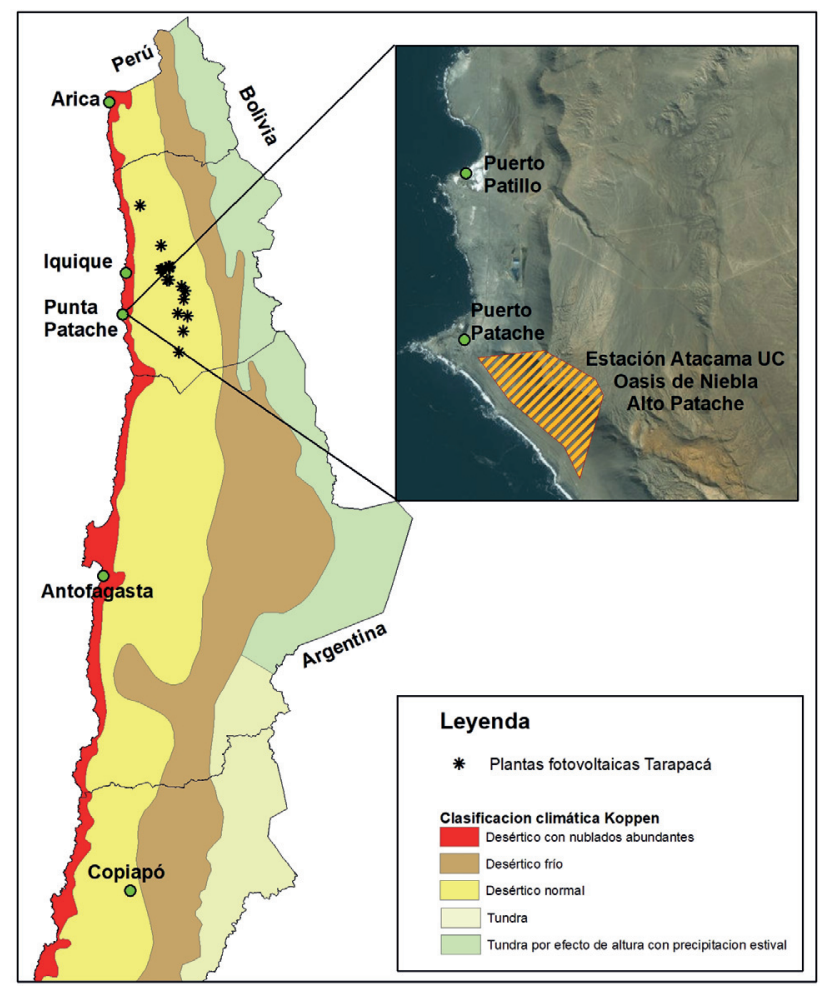

Fuente: Elaboración propia.

\section{Materiales y métodos}

A continuación, se describen los pasos e instrumentos utilizados en la obtención y procesamiento de datos para la caracterización de presencia/ausencia de nube de estratocúmulo y niebla, porcentaje de cobertura y tipología de niebla, volúmenes de captación de agua-niebla $\left(\mathrm{L} \mathrm{m} \mathrm{m}^{2}\right.$ día $^{-1}$ ) y comportamiento de la radiación solar (irradiancia $\mathrm{W} / \mathrm{m}^{2}$ ). Se trabajó el mes de septiembre 2013 por ser el mes de mayor captación de agua de niebla (Larraín et al., 2002; Cereceda et al., 2008b; Del Río et al., 2017) y el año 2013 por ser un año promedio dentro de los últimos 20 años en cuanto a los rendimientos de agua de niebla colectados en Alto Patache para dicho mes (Del Río et al., 2017).

\section{Caracterización nube estratocúmulo}

Mediante la aplicación de técnicas de teledetección se caracterizó la dinámica diaria de la nube estratocúmulo (Sc) y a su vez se determinaron el porcentaje de cobertura nubosa y las tipologías de niebla presentes en la costa de Tarapacá, específicamente sobre el sector de Alto Patache. 
Para el análisis espaciotemporal de la nube Sc, se procesaron las imágenes satelitales del sensor geoestacionario GOES-13 para el intervalo de longitud entre -71.0000 a -69.0000 y las latitudes -19.0000; -21.5000 (Figura $N^{\circ} 2$ ), disponibles para septiembre de 2013. Se procesaron un total de 100 imágenes correspondiente a 20 días, que son las utilizables excluyendo días sin disponibilidad de imágenes o con presencia de nubosidad alta. Se analizaron 5 imágenes por día, capturadas a las 04:39, 07:39, 10:39, 15:39 y 21:39 UTC (01:39, 04:39, 07:39, 12:39 y 19:39 hora local respectivamente) que corresponden a los umbrales diarios de máxima (noche) y mínima (medio día) presencia de niebla, junto a las inflexiones de presencia de niebla al amanecer y atardecer (Farías et al., 2005; Cereceda et al., 2008a). La identificación de las nubes bajas en horarios nocturnos se realizó con la técnica basada en la diferencia entre las longitudes de onda del infrarrojo térmico corto $(3,8 \mu \mathrm{m})$ y largo $(10,9 \mu \mathrm{m})$ (Eyre et al., 1984; Ellrod, 1995; Anthis \& Cracknell, 1999; Bendix 2002; Underwood et al., 2004, del Río et al., 2017). Para los horarios diurnos se aplicaron técnicas de detección de cambios utilizando el rango visible, y para discriminar entre nubes bajas y altas el rango térmico $(10,9 \mu \mathrm{m})$ (Jedlovec \& Laws, 2003; Torregrosa et al., 2015). Una vez identificadas las nubes bajas se procede a determinar: 1) la presencia o ausencia de nubes bajas (nube Sc o niebla según corresponda); 2) Estimar el porcentaje de cobertura nubosa para un área circular de 200 km² sobre la Estación Atacama UC Alto Patache; 3) Determinar visualmente la tipología de niebla presente, entre advectiva y orográfica (Figura $\mathrm{N}^{\circ} 2$ ).

Figura $\mathrm{N}^{\circ} 2$

Clasificación e identificación de nubes bajas a partir de imágenes del sensor GOES-13 para el 27 de septiembre de 2013, 18:39 hora local. El círculo rojo corresponde al área sobre la que se estima la cobertura nubosa sobre Alto Patache. En la imagen se observa el caso de niebla orográfica

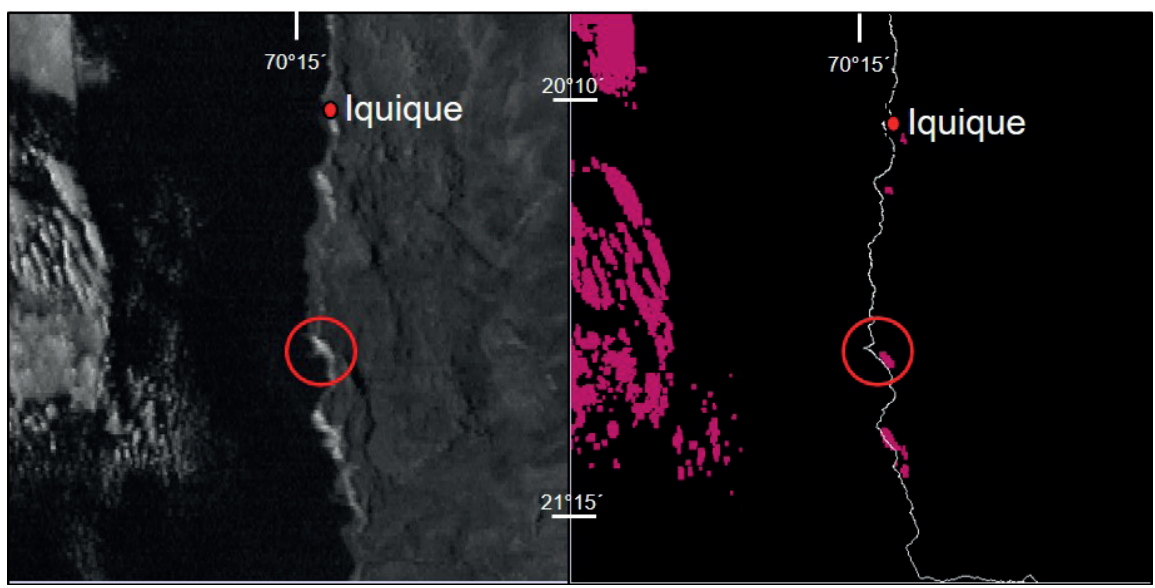

Fuente: Elaboración propia.

\section{Captación de agua de niebla}

Para cuantificar los volúmenes de agua que se obtienen y determinar el comportamiento temporal de la captación de agua de niebla, se utilizó un neblinómetro estándar o standard fog collector (SFC) de L m² (Schemenauer \& Cereceda, 1994) (Figura N³), este captador pasivo funciona 
en base a una malla sombreadora tipo "raschel" de 35\% sombra puesta en dos capas a una altura de dos metros en su base, el agua es almacenada en un estanque dispuesto para tales efectos, el cual es controlado con periodicidad constante. En Alto Patache se ha medido ininterrumpidamente la colección de agua desde el año 1997 en una base mensual principalmente.

Figura $\mathrm{N}^{\circ} 3$

Neblinometros (Standard Fog Collector SFC) instalados en Alto Patache. Ubicación: sobre acantilado costero en Estación Atacama UC, al fondo se visualiza estación meteorológica 850 m s.n.m.)

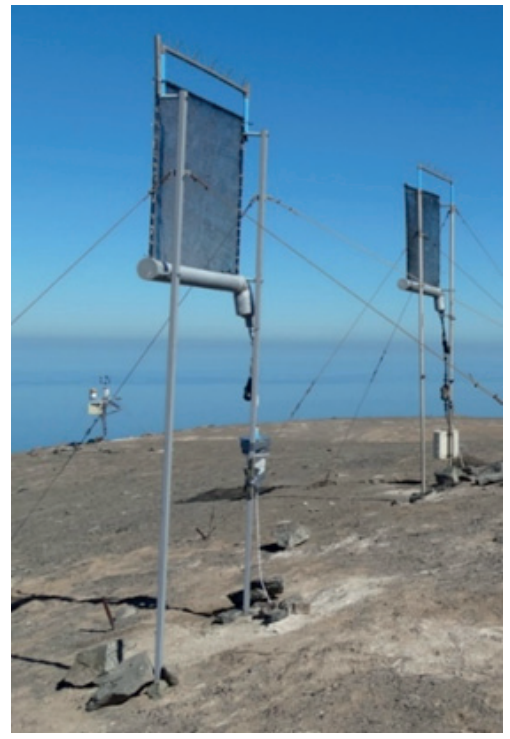

Fuente: Elaboración propia.

\section{Energía solar - Irradiancia}

La irradiancia solar en Alto Patache se mide desde enero de 2013 con un sistema del tipo "Rotating Shadowband Radiometer" (RSBR), o radiómetro de banda de sombreamiento (Figura $\mathrm{N}^{\circ} 4$ ). La configuración básica para este tipo de dispositivos incluye un radiómetro fotoeléctrico LI-200, un controlador de motor y una banda de sombreamiento rotativa, sensores de temperatura, de presión atmosférica, humedad relativa, todos conectados a un datalogger, con fuente de alimentación de un sistema fotovoltaico de pequeña escala (Escobar et al., 2015).

La banda de sombreamiento rota alrededor del sensor y genera una sombra periódica cada 1 minuto. En el momento del sombreado el sensor Licor mide irradiancia difusa horizontal (DHI), y calcula la irradiancia directa horizontal como la resta de global menos difusa.

Por otra parte, la latitud del sitio precisa la trayectoria del sol en el cielo, que define el ángulo de elevación solar. Este ángulo se utiliza para calcular la radiación directa normal (DNI, Direct Normal Irradiance) a partir de la radiación directa horizontal, y de esta forma el RSBR puede obtener las tres componentes principales de la radiación solar a partir de mediciones realizadas con un sensor único. 
Figura $\mathrm{N}^{\circ} 4$

Sistema RSBR instalado en Alto Patache al borde del acantilado costero, a una altitud de 798 m s.n.m.

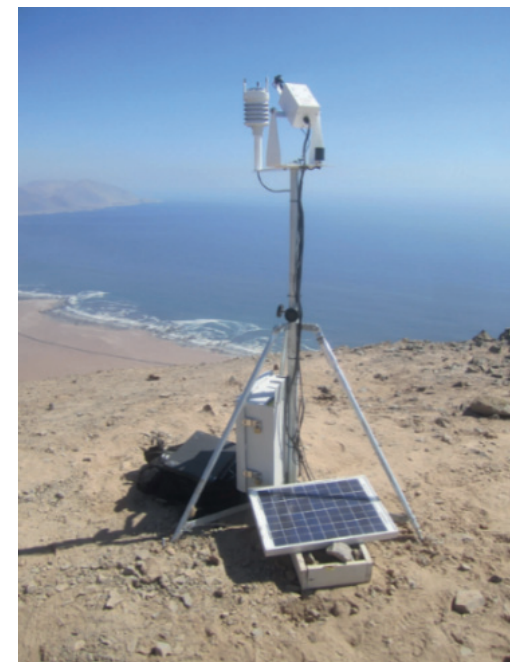

Fuente: Elaboración propia.

El sistema de medición RSBR se opera de acuerdo a criterios internacionales de operación y mantenimiento (Wilbert et al., 2015) y produce datos de calidad controlada que son válidos para aplicaciones de tecnologías de energía solar y caracterización meteorológica (ISO:9060, 1990). Los certificados de calibración del piranómetro se mantienen actualizados y trazados directamente a la referencia mundial a través de calibración en laboratorio y comparaciones en terreno con pirheliómetros participantes en la International Pyrheliometer Comparison IPC XIII realizada en 2015 en Davos.

Es posible evaluar la incertidumbre asociada a las mediciones en terreno del RSR al considerar el tipo de sensor, su estado de calibración, respuesta al ángulo cenital, respuesta espectral, no-linealidad en la respuesta a la temperatura, degradación anual, precisión del sistema de adquisición de datos, y condiciones de operación y mantenimiento (Habte et al., 2014). La incertidumbre de los resultados se calcula usando la metodología GUM (STANDARD:SAUDI, 2006) para el sensor fotoeléctrico, además de comparaciones en laboratorio y terreno para GHI y DNI medidos con sensores de estándar secundario (ISO:9060, 1990).

\section{Pérdida de radiación solar por efecto de la nube local}

Existen modelos que estiman la DNI que se registraría si el cielo estuviese despejado, el modelo de DNI de cielo claro más utilizado y validado es el de Bird (Bird \& Hulstrom, 1981). Usando este modelo en comparación a la DNI real registrada se puede identificar la presencia de atenuantes atmosféricos. Así un día completamente claro tiene una distribución diaria de DNI cercana a la DNI señalada por Bird, mientras que la DNI de un día nublado es significativamente menor a la dada por el modelo de Bird. 
Finalmente, para integrar las distintas bases de datos analizadas en el presente artículo se consideraron las siguientes homologaciones horarias (Cuadro $N^{\circ} 1$ ).

\section{Cuadro $\mathrm{N}^{\circ} 1$}

Homologación horaria de datos analizados

\begin{tabular}{|l|c|c|c|c|c|}
\hline & \multicolumn{5}{|c|}{ Septiembre 2013} \\
\hline Hora (UTC) Imágenes satelitales GOES & $04: 39$ & $07: 39$ & $10: 39$ & $15: 39$ & $21: 39$ \\
\hline Hora Local Imágenes GOES & $01: 39$ & $04: 39$ & $07: 39$ & $12: 39$ & $18: 39$ \\
\hline $\begin{array}{l}\text { Homologación horaria con datos de radiación y } \\
\text { agua de niebla }\end{array}$ & $02: 00$ & $05: 00$ & $08: 00$ & $13: 00$ & $19: 00$ \\
\hline
\end{tabular}

Fuente: Elaboración propia.

\section{Comportamiento de la irradiancia y cobertura de niebla en Alto Patache}

Cuando se trabaja a escala anual las variables de irradiancia y agua de niebla, resulta interesante observar que ambas presentan una curva característica con periodos de baja y alta disponibilidad relativa. En el caso de la energía solar esta obviamente cambia con la declinación del sol en periodos invernales y estivales para menores y mayores productividades respectivamente. A diferencia del recurso hídrico que tiene su periodo de mayor disponibilidad en invierno y primavera, con una marcada baja durante el verano y principios del otoño, como se puede observar en la Figura $\mathrm{N}^{\circ} 5$. Asimismo, se observa que el recurso hídrico, que se encuentra asociado a nubosidad abundante por ejemplo en septiembre, no es coincidente con los mínimos valores de irradiancia en Alto Patache, siendo entonces la estacionalidad astronómica aparentemente más relevante que la presencia de nubosidad, especialmente en zonas donde esta última está muy presente.

La cobertura nubosa analizada (Sc) para septiembre de 2013 tiene presencia sobre Alto Patache (Figura $\mathrm{N}^{\circ}$ 2) en un $78 \%$ de los horarios analizados, con un ciclo diario que muestra las máximas coberturas durante la noche llegando al $80 \%$ (02:00 y 05:00 horas), baja durante el amanecer y al medio día (65\% a las 08:00 y 13:00 horas) y llega a un 100\% de presencia al atardecer (19:00 horas).

Por otra parte, al analizar el porcentaje de cobertura en presencia de niebla en el área estudiada, durante la noche alcanza un $92 \%$, al amanecer llega a un $70 \%$, al medio día baja a un $58 \%$ y al atardecer llega sólo a un $38 \%$.

Si el mismo análisis se repite, pero esta vez distinguiendo por tipología de niebla, encontramos que, del total de horas trabajadas, el 58\% correspondió a eventos advectivos, un $20 \%$ a orográficos y un $22 \%$ no presentaron cobertura. Al diferenciarlos por horarios y sólo en aquellos con presencia de alguna tipología de niebla, se observa que durante la noche el $94 \%$ y el $100 \%$ corresponden a eventos advectivos (02:00 y 05:00 horas respectivamente), al amanecer hay un 
Figura $\mathrm{N}^{\circ} 5$

Variabilidad anual de captación de agua de niebla $(\mathrm{L})$ y radiación solar $\left(\mathrm{W} / \mathrm{m}^{2}\right)$. Año de estudio 2013

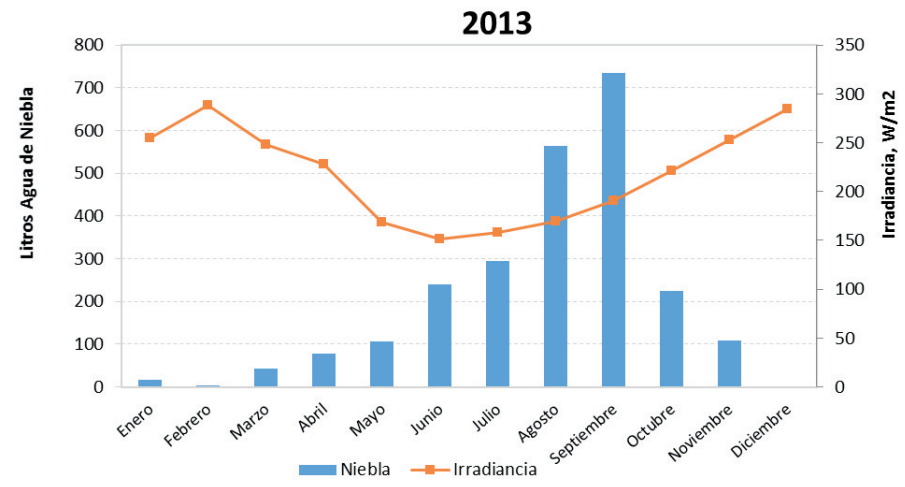

Fuente: Elaboración propia.

$100 \%$ de presencia de niebla advectiva, lo cual varía al mediodía, pasando a un $54 \%$ de advectivas versus $46 \%$ de orográficas, para finalmente al atardecer tener un $35 \%$ de advectivas versus $65 \%$ de orográficas.

En relación con la cobertura que presentan las distintas tipologías, encontramos que las advectivas durante la noche cubren en promedio un $94 \%$ del área analizada respecto a un $40 \%$ del único evento orográfico nocturno observado. Al amanecer, sólo se identificaron nieblas advectivas las que cubren un $70 \%$ del área. Al mediodía nieblas también advectivas cubren un $80 \%$ versus un $32 \%$ de las orográficas, mientras que al atardecer las advectivas alcanzan un $77 \%$ versus el $17 \%$ de las orográficas.

La Figura $N^{\circ} 6$ integra la irradiancia y el porcentaje de cobertura nubosa para el periodo de tiempo analizado, el cual muestra, además de los ciclos diarios de ambos indicadores, que en presencia de un mayor porcentaje de cobertura la irradiancia disminuye, pero dado el comportamiento horario descrito (nubosidad predominante en la noche), la relación general es inversa (correlación: $(-0,452)$ con un $\mathrm{p}=0,001)$ lo que da cuenta de que en primera instancia no son recursos que se anulen al mismo tiempo cuando comparten un territorio. Podemos además señalar que la variabilidad en irradiancia responde de diferente manera al porcentaje de cobertura nubosa y sus tipologías.

Al omitir los horarios nocturnos, donde no hay irradiancia solar, se observa que el promedio es de $170 \mathrm{~W} / \mathrm{m}^{2}$ en presencia de eventos advectivos y de $240 \mathrm{~W} / \mathrm{m}^{2}$ como promedio para los eventos orográficos, mostrando que durante eventos advectivos la irradiancia es casi 30\% menor. La Figura $N^{\circ} 7$ muestra que las mayores atenuaciones están relacionadas con eventos advectivos. Este comportamiento se explica en función de las características de las nieblas orográficas que son más parciales en su cobertura espacial alternando nube y cielo despejado, permitiendo que exista ingreso de radiación con mayor frecuencia durante la ocurrencia de estos eventos respecto a los advectivos en que la cobertura es más homogénea. 
Figura $N^{\circ} 6$

Comportamiento de la Irradiancia $\left(\mathrm{W} / \mathrm{m}^{2}\right)$ y cobertura de niebla (\%) a las $02.00-05.00-08.00-13.00$ y 19.00 horas para el mes de septiembre 2013

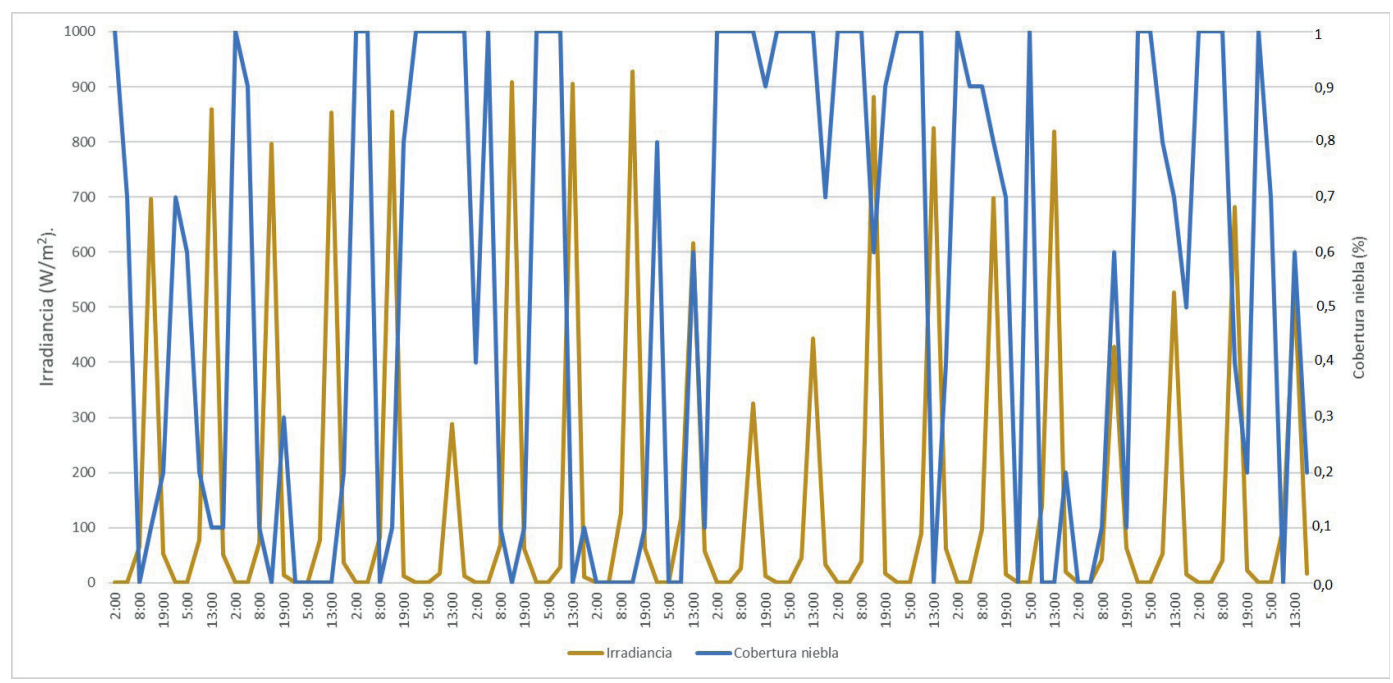

Fuente: Elaboración propia.

Figura N07

Relación entre la irradiancia solar y tipología de evento de niebla

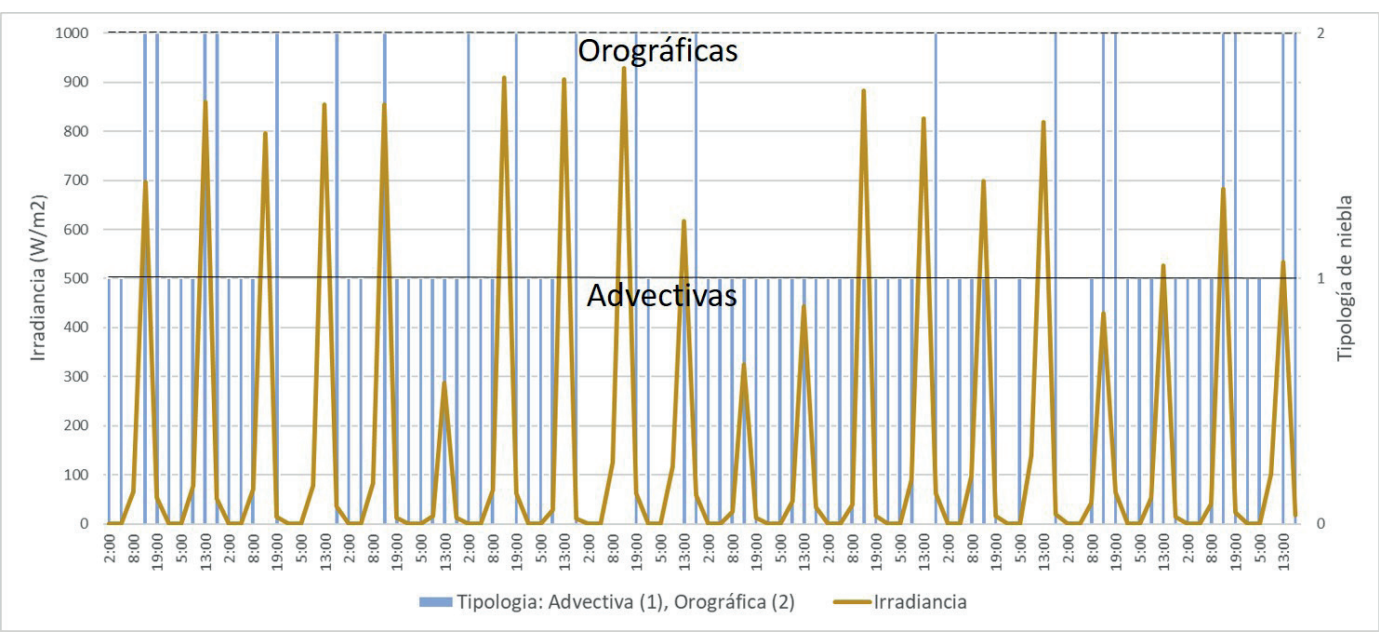

Fuente: Elaboración propia.

Al calcular los promedios de irradiancia por tipología de niebla para los horarios diurnos (Figura N08), encontramos que para las 08:00 horas es de $55 \mathrm{~W} / \mathrm{m}^{2}$ y en este caso sólo hay presencia de eventos advectivos. Para el mediodía la irradiancia promedio de $539 \mathrm{~W} / \mathrm{m}^{2}$ bajo eventos advectivos y un promedio de $675 \mathrm{~W} / \mathrm{m}^{2}$ durante nieblas orográficas, mostrando una diferencia de 
atenuación de 25\% menor en el caso orográfico. Finalmente, al atardecer (19:00 horas) la irradiancia promedio cambia de $16 \mathrm{~W} / \mathrm{m}^{2}$ en eventos advectivos versus más de $40 \mathrm{~W} / \mathrm{m}^{2}$ durante eventos orográficos, demostrando una atenuación $156 \%$ menor. Como se puede observar en el grafico que corresponde a las 13:00 (Figura $N^{\circ} 8$ ), que presenta los mínimos de irradiancia en condiciones de niebla advectivas, como por ejemplo los días 12 y 21 del mes estudiado (septiembre 2013). Asimis-

Figura $\mathrm{N}^{\circ} 8$

Atenuación de la irradiación por tipología de niebla y horario

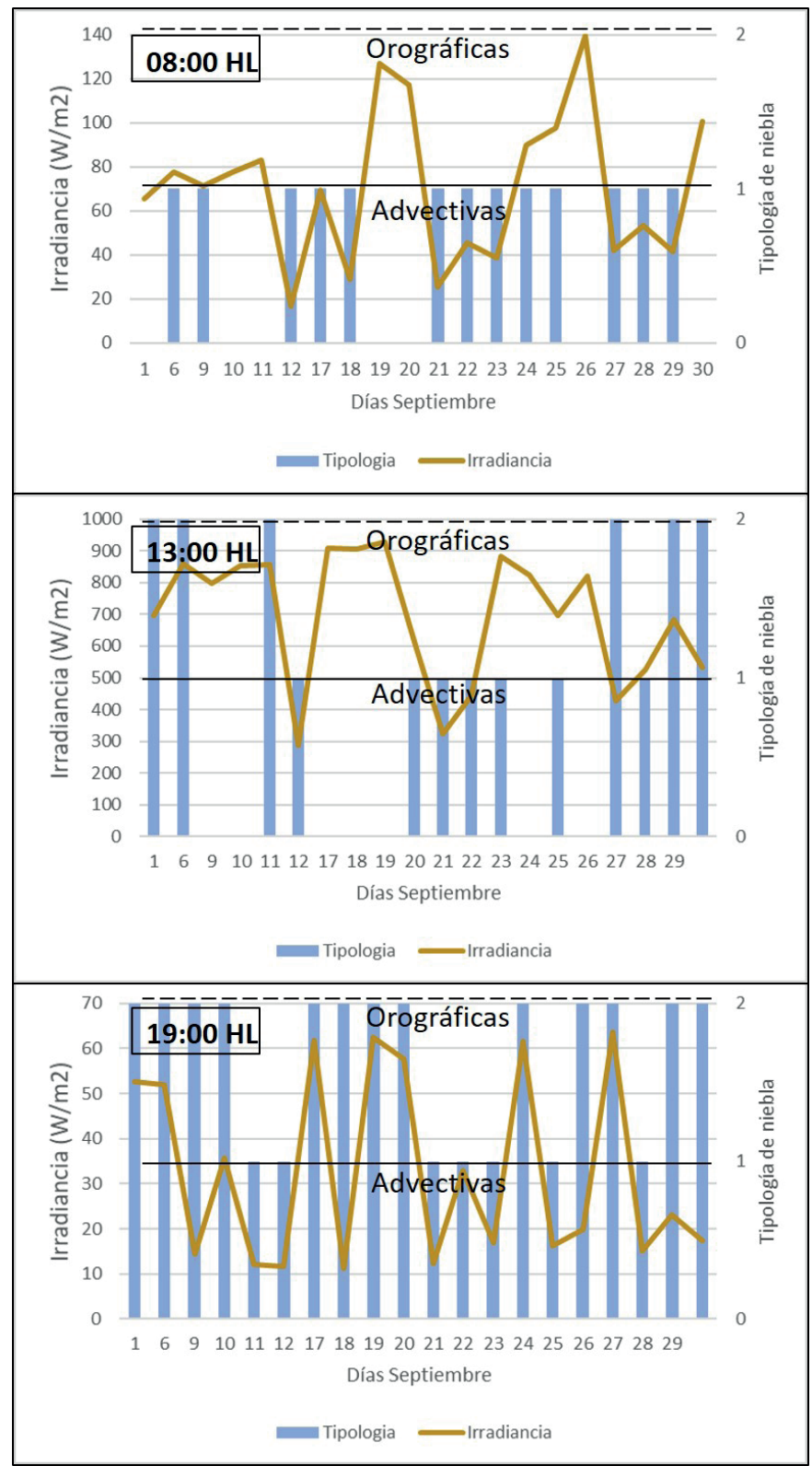

Fuente: Elaboración propia. 
mo, la máxima irradiancia en presencia de niebla, se dan en condiciones orográficas como se observa en el gráfico correspondiente a las 19:00 horas de los días 17, 19, 24 y 27 de septiembre 2013.

Lo anteriormente señalado coincide con la dinámica física de una y otra tipología de niebla, en que los fenómenos advectivos son más homogéneos en su cobertura espacial respecto de aquellos orográficos que son más dinámicos y tienen alternancia de nubosidad y espacios despejados, especialmente durante sus inicios y términos. Por otro lado, el grosor y densidad de los eventos advectivos es mayor en relación a aquellos de origen orográfico.

Al analizar los rendimientos y cantidades de agua de niebla colectada por horario y por tipología (advectiva y orográfica) se observa que los eventos advectivos tienen asociado más productividad de agua, tal es el caso por ejemplo del 28 de septiembre que presenta sólo eventos advectivos en los cinco horarios analizados y que se recolectan (112 $\left.1 \mathrm{~m} \mathrm{~m}^{-2} \mathrm{dí}^{-1}\right)$, siendo un $72 \%$ superior a aquellos días con nieblas principalmente orográficas y $95 \%$ más que días con sólo eventos orográficos al mediodía y atardecer. Esta situación se asocia con las características de cada tipología de niebla, donde el evento advectivo es de mayor duración, generalmente desde horas de la tarde, toda la noche hasta la madrugada y con una cubierta de nube más homogénea y completa (Cereceda et al., 2002). Distinto de la condición orográfica que ocurre en horas de la tarde disipándose en la noche, asimismo su cobertura no es necesariamente homogénea como ya se ha señalado.

\section{Conclusiones}

El clima "desértico costero con nublados abundantes" del desierto de Atacama, presenta dos recursos naturales relevantes y complementarios como son la radiación solar y el agua de niebla. La generación de energía y la disponibilidad de agua son recursos de creciente demanda en estos territorios, los cuales buscan opciones sustentables para su desarrollo presente y futuro.

El ciclo diario de la nubosidad predominante muestra presencia en un $78 \%$ de los horarios trabajados, con una alta frecuencia en los atardeceres y noches (100\% y $80 \%$ respectivamente) y levemente en menor medida durante la madrugada (70\%), momentos en que hay total ausencia de irradiancia solar por motivos astronómicos. Lo opuesto ocurre durante la mayor parte del día, donde se presenta una importante disminución de la nubosidad, (menos de $60 \%$ de presencia), lo que da paso a una irradiancia que alcanza como promedio los $862 \mathrm{~W} / \mathrm{m}^{2}$ en condiciones de cielo claro. Esto nos lleva a dar cuenta de que las nieblas costeras y la captación de agua que se hace de ellas constituyen un recurso hídrico concreto, situación que ya ha sido documentada con creces. Asimismo, el recurso energético solar se ve respaldado por los valores de irradiancia registrados y presentados en este trabajo, podemos entonces concluir que no existe una anulación entre ambos recursos (agua y energía) más bien podemos referirnos a una marcada complementariedad en sus ciclos diarios.

Más aún, las tipologías de nieblas presentes reafirman que no hay traslape dado que las nieblas advectivas, tienen mayor presencia en los horarios nocturnos (01:00, 05:00 y 08:00 horas) y a su vez son las que tienen una mayor cobertura espacial, mientras que las de tipo orográficas tienen porcentualmente mayor presencia en horarios diurnos (13:00 y 19:00) y su cobertura espacial resulta muy puntual y acotada, momento en que la radiación solar es mayor. Asimismo, son 
las nieblas advectivas las que tienen una mayor productividad de agua respecto a los eventos orográficos, con diferencias de más del $80 \%$ entre ambas tipologías.

Se establece en este trabajo la complementariedad entre el recurso hídrico de niebla y la generación y disponibilidad de energía a partir del recurso solar. Más aún permite reflexionar sobre una potencial integración productiva de ambas fuentes, como podría ser la distribución de agua de niebla con energía solar hacia territorios que no disponen las condiciones para captar éste recurso hídrico alternativo, aumentar la eficiencia de captación del agua de niebla a través del enfriamiento de las redes de captación para aumentar la condensación, la utilización del agua de niebla para la limpieza del polvo que se acumula en los paneles solares y que disminuyen significativamente la producción de energía, tratamiento y mantención de la calidad del agua, especialmente pensando en la alta producción de agua durante invierno y primavera, en contraposición con el verano y parte del otoño, lo que lleva a proponer el aprovechamiento de la energía disponible para la implementación de mecanismos de mantención de las propiedades del agua durante períodos de tiempo más prolongados. En este sentido es que resulta fundamental la obtención, mantención y perfeccionamiento de las series de datos de irradiancia, agua de niebla y cobertura nubosa para seguir explorando estos recursos necesarios y de creciente demanda en territorios desérticos especialmente.

La disponibilidad de un sitio de investigación como la Estación Atacama UC por su localización, equipamiento y generación de nueva información en los últimos veinte años, resulta ser de gran interés científico, para diversas aplicaciones, tanto en el área de geografía como en ciencias afines para lograr trabajos multidisciplinarios, el cual se recomienda conservar y monitorear en el tiempo, así por ejemplo ya se realizan iniciativas de bombeo de agua mediante sistemas alimentados por energía solar, investigación y desarrollo de especies vegetales así como también técnicas de construcción y de-construcción menos invasivas, siempre pensando en ambientes desérticos y en las tendencias climáticas actuales

\section{Referencias bibliográficas}

AGOSTINI, C.; SILVA, C. \& NASIROV, S. Solar Energy in Chile: Development and Challenges. Revista Harvard Review of Latin America, 2015, Vol. 15, №1, p. 40-42.

ANTHIS, A. \& CRACKNELL, A.P. Use of satellite images for fog detection (AVHRR) and forecast of fog dissipation (METEOSAT) over lowland Thessalia, Hellas. Int J Remote Sens, 1999, Vol. 20, N 6, p. 1107-1124.

BANCO MUNDIAL. Chile. Diagnóstico de la gestión de los recursos hídricos. Departamento de Medio Ambiente y Desarrollo Sostenible, Región para América Latina y el Caribe, 2011, p. 81.

BENDIX, J. A satellite-based climatology of fog and low level stratus in Germany and adjacent areas. Atmos, 2002, Vol. 64, № 1-4, p. 3-18.

BIRD, R.E. \& HULSTROM, R.L. A Simplified Clear Sky Model for Direct and Diffuse Insolation on Horizontal Surfaces. SERI/TR-642-761. Golden, CO: Solar Energy Research Institute, 1981. 
CÁCERES, L.; GÓMEZ-SILVA, B.; GARRÓ, X.; RODRÍGUEZ, V.; MONARDES, V. \& MCKAY C.P. Relative humidity patterns and fog water precipitation in the Atacama Desert and biological implications, Journal of Geophysical Research, 2007, Vol. 112, № G4. Disponible en internet: http://onlinelibrary. wiley.com/doi/10.1029/2006JG000344/pdf

CENTRO DE INVESTIGACIÓN Y DESARROLLO EN RECURSOS HÍDRICOS, CIDERH. Recursos hídricos Región de Tarapacá. Iquique: Ediciones Universidad Arturo Prat, 2013.

CERECEDA, P.; OSSES, P.; LARRAÍN, H.; FARÍAS, M.; LAGOS, M.; PINTO, R. \& SCHEMENAUER, R.S. Advective, orographic and radiation fog in the Tarapacá region, Chile. Atmospheric Research, $2002, N^{\circ} 64$, p. 261-271.

CERECEDA P.; LARRAIIN, H.; OSSES, P.; FARÍAS, M. \& EGAÑA, I. The climate of the coast and fog zone in the Atacama Desert of Tarapacá Region, Chile. Atmospheric Research, 2008a, № 87, p. 301-311.

CERECEDA P.; LARRAIN H.; OSSES P.; FARÍAS M. \& EGAÑA I. The spatial and temporal variability of fog and its relation to fog oases in the Atacama Desert, Chile. Atmospheric Research, 2008b, $\mathrm{N}^{\circ}$ 87, p. 312-323.

CNE/PNUD/UTFSM. Irradiancia solar en territorios de la República de Chile. Santiago de Chile: Registro solarimétrico, 2008, p. 248.

DEL RÍO, C.; GARCIA, J.L.; OSSES, P.; ZANETTA, N.; LAMBERT, F.; RIVERA, D.; SIEGMUND, A.; WOLF, N.; CERECEDA, P.; LARRAÍN, H. \& LOBOS, F. ENSO influence on coastal fog-water yield in the Atacama Desert, Chile. Aerosol and Air Quality Research, 2017. DOI: 10.4209/aaqr.2017.01.0022

DIRECCIÓN METEOROLÓGICA DE CHILE. Informe de precipitaciones. 2017. Disponible en Internet: http://www.meteochile.gob.cl/PortalDMC-web/index.xhtml

ELLROD, G. Advances in the Detection and Analysis of Fog at Night Using GOES Multiespectral Infrared Imagery. Weather \& Forecasting, 1995, N¹0, p. 606-619.

ESCOBAR, R.; CORTÉS, C.; PINO, A.; SALGADO, M.; PEREIRA, E.; RAMOS-MARTINS, F.; BOLAND, J. \& CARDEMIL, J. Estimating the potential for solar energy utilization in Chile by satellite-derived data and ground station measurements. Solar Energy, 2015, Vol. 121, p. 139-151.

EYRE, J.R. (Detection of fog at night using Advanced Resolution Radiometer (AVHRR) imagery. Meteor. Mag., 1984, Nº113, p. 266-271.

FARÍAS, M.; CERECEDA, P.; OSSES, P. y NÚÑEZ, R. Comportamiento espacio-temporal de la nube estratocúmulo, productora de nieva en la costa del desierto de Atacama (21이. S., $70^{\circ}$ long. W.), durante un mes de invierno y otro de verano. Investigaciones Geográficas, Boletín del Instituto de Geografía, UNAM, 2005, N 56, p. 43-61. 
HABTE, A.; LOPEZ, A.; SENGUPTA, M. \& WILCOX, S. Temporal and spatial comparison of gridded TMY, TDY, and TGY data sets. National renewable energy laboratory, U.S. Department of Energy, 2014. Disponible en internet: https://www.nrel.gov/docs/fy14osti/60886.pdf

ISO 9060:1990 Standard: Solar energy -- Specification and classification of instruments for measuring hemispherical solar and direct solar radiation.

JEDLOVEC, G.J. \& LAWS, K. Goes cloud detection at the global hydrology and climate center. In: $12^{\text {th }}$ Conference on satellite meteorology and oceanography, poster session 1, Climatology and clouds, 2003.

KLEISSL, J. Solar energy forecasting and resource assessment. Editorial Academic press, 2013.

KLEMM, O.; SCHEMENAUER, R.S.; LUMMERICH, A.; CERECEDA, P.; MARZOL, V.; CORELL, D.; VAN HEERDEN, J.; REINHARD, D.; GHEREZGHIHER, T.; OLIVIER, J.; OSSES, P.; SARSOUR, J.; FROST, E.; ESTRELA, M.J.; VALIENTE, J.A. \& FESSEHAYE, G.M. Fog as a Fresh-Water Resource: Overview and Perspectives. Ambio, 2012, N 41, p. 221-234.

LARRAÍN, H.; VELÁSQUEZ, F.; CERECEDA, P.; ESPEJO, R.; PINTO, R.; OSSES P. \& SCHEMENAUER R. $S$. Fog measurements at the site "Falda Verde" north of Chañaral compared with other fog stations of Chile. Atmospheric Research, 2002, N 64, p. 273-284.

ORTEGA, A.; ESCOBAR, R.; COLLE, S. \& LUNA DE ABREU, S. The state of solar energy resource assessment in Chile. Renewable Energy, 2010, Vol. 35, No 11, p. 2514-2524.

OSSES, P.; BARRÍA, C.; FARÍAS, M. y CERECEDA, P. La nube estratocúmulo en Tarapacá, Chile. Validación de imágenes GOES mediante observación en tiempo real (17 al 26 de julio del año 2002). Revista de Geografía de Norte Grande, 2005, №33, p. 131-143.

PARRADO, C.; GIRARD, A.; SIMON, F. \& FUENTEALBA, E. 2050 LCOE (Levelized Cost of Energy) projection for a hybrid PV (photovoltaic)-CSP (concentrated solar power) plant in the Atacama Desert, Chile. Energy, 2016, Nº 94, p. 422-430.

SANTANA, C.; FALVEY, M.; IBARRA, M. y GARCÍA, M. Energías renovables en Chile. El potencial eólico, solar e hidroeléctrico de Arica a Chiloé. Proyecto Estrategia de Expansión de las Energías Renovables en los sistemas eléctricos interconectados. Santiago de Chile: Ministerio de Energía/ GIZ, 2014. Disponible em internet:

http://www.minenergia.cl/archivos_bajar/Estudios/Potencial_ER_en_Chile_AC.pdf

SARMIENTO, P. Energía solar. Aplicaciones e ingeniería. Valparaíso: Ediciones universitárias de Valparaíso, Pontificia Universidad Católica de Valparaíso, 2009.

SCHEMENAUER, R. \& CERECEDA, P. Fog collection's role in water planning for developing countries. Natural resources forum ONU, 1994, Vol. 18, N² 2, p. 91-100. 
STANDARD:SAUDI. Draft NO. 13/2000. Guide to the expression of uncertainty in measurement. Riad: Saudi Arabian standards organization, 2006.

TORREGROSA, A.; COMBS, C. \& PETERS, J. GOES-derived fog and low cloud indices for coastal north and central California ecological analyses. Earth and Space Science, 2016, Vol. 3, No 2. Disponible en internet:

http://onlinelibrary.wiley.com/doi/10.1002/2015EA000119/pdf

UNDERWOOD, J.; ELLROD, G. \& KUHNERT, A. A multiple-case analysis of nocturnal radiation-fog development in the central valley of California utilizing the GOES nighttime fog product. Journal of Applied Meteorology and Climatology, 2004, № 43, p. 297-311.

WEISCHET, W. Las condiciones climáticas del desierto de Atacama como desierto extremo de la tierra. Revista Norte Grande, 1975, Vol. 1., №3 - 4, p. 363-373.

WILBERT, S.; GEUDER, N.; SCHWANDT, M.; KRAAS, B.; JESSEN, W.; MEYER, R. \& NOURI, B. Best practices for solar irradiance measurements with rotating shadowband irradiometers. Solar Heating \& Cooling Programme, International Energy Agency, 2015. Disponible en internet: http://task46.iea-shc.org/data/sites/1/publications/INSRSI_IEA-Task46B1_BestPractices-RSI_150819.pdf 